\title{
Dressings For Burn Injury In A Military Conflict - Change Of Practice Based On Current Evidence
}

\author{
T La H Brown
}

\begin{abstract}
Introduction
Conventional burn dressings aim to protect the injury from microbiological invasion, contribute significantly to pain relief, and protect healthcare staff from infective risks. Acutely, cling film is employed successfully, as described by many of the advertisements for the TA in the weekly medical press. Although providing good analgesia and facilitating regular inspection of the injury without exposure, it is only a temporary measure and provides no protection from infection with burn exudate accumulating rapidly beneath the film. When used as the only dressing, patients become rapidly hypothermic.

When there is no NBC threat, the burn wound takes a lower priority to stabilization of the patient. One the airway and appropriate fluid management are instituted, the burns should be washed in warm soapy water and any loose tissues or blisters removed. The goal of subsequent care is to control the development of invasive infection until the wound heals spontaneously or as a result of surgery.
\end{abstract}

\section{Topical anti microbial applications}

Burned skin has a compromised blood supply and is prone to invasion by microbial organisms. Initially, a burn is sterile, but rapidly becomes colonised, predominantly by gram positive cocci in the first instance. As the bacterial density increases there is spread through the eschar by migration along the sweat ducts. Spread is unimpaired due to a poor blood supply and a compromised immune response which is also a feature of thermal injury. Once bacterial density reached $10^{5} / \mathrm{g}$ of tissue wound infection and septicaemia are likely to occur. Early application of topical anti microbial agents is the most effective way of preventing invasive burn infection when combined with early excision of the eschar.

Although topical antimicrobial agents have been shown to confer a survival benefit when applied to burns (1), there have been few comparative trials of one against another, as each fills a particular niche with its own complications and benefits.

\section{Silver nitrate}

Silver nitrate solution $(0.5 \%)$ has been used since the 1800 s as an agent with the ability to 'tan' eschar and prevent bacterial penetration. It is, however, unpleasant to handle because it discolours everything on contact and dressing require regular soaking to prevent concentrations from rising to cytotoxic levels with water evaporation. Trans eschar leaching of electrolytes occurs from the circulation, becoming significant when applied over a large surface area necessitating intravenous supplementation. As silver nitrate precipitates protein on contact to form a 'tan' layer, deeper penetration into the burn eschar is prevented. For this reason it is only suitable for immediate treatment of burns and is ineffective in established wound infection. Recently, a nano crystalline silver nitrate impregnated absorbant dressing

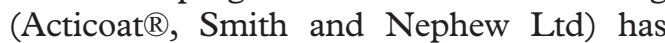
become available, which can be applied directly to a fresh burn and left in place for up to 5 days without attention. The spectrum of microbiological resistance to this material is at present very small. Although it has only been introduced in the UK recently, it is used extensively in the USA with success, and may be useful in conflict environments.

\section{Silver sulphadiazene (Flamazine)}

Silver sulphadiazene burn cream $(1 \%$ in a water soluble base, Flamazine, Smith and Nephew Ltd) is a popular topical application because in addition to its microbiological properties, it is soothing, and does not leach sodium significantly. It is currently the recommended application to burn injury in the British Armed Forces. However, Flamazine requires re-application every 24 hours in a layer at least $1 / 8$ th inch thick to be effective. As a rule of thumb, an estimate $10 \mathrm{~g}$ silver sulphadiazene per percent burn per 24 hours are required or one $400 \mathrm{~g}$ tub per day for a $40 \%$ burn. Like silver nitrate, it has no ability to penetrate eschar. It is ineffective in established burn infection and only of value on a fresh burn. It suffers from a multi resistance problem, particularly to pseudomonas and enterobacter (1). Exclusive use of silver sulphasalazine as a topical anti microbial is responsible for the spread of plasmid bourne multi resistance to antibiotics. MRSA have been found to grow in tubs of silver sulphadiazine opened for more than 24 hours and these should, therefore, be discarded after initial use (2). Neutropenia is reported with regular application of silver sulphadiazine 
during the first five days following injury, although blood counts return to normal on cessation of use.

\section{Mafenide acetate (Sulfamylon)}

Mafenide acetate (Sulfamylon, Bertex Pharmaceutical Inc.) can be applied as a topical $5 \%$ solution, made up with tap water. It has limited problems with bacterial resistance, but importantly none to pseudomonas the commonest speticaemia in burn deaths (1). Mafenide cream has been referred to by patients as 'white lightening' due to the discomfort when applied to partial thickness. There is a $7 \%$ incidence of sensitivity, producing an erythematous rash which can be treated by either stopping therapy or oral antihistamines. More seriously, mafenide is an inhibitor of carbonic anhydrase, producing a bicarbonate diuresis and accentuating post-burn hyperventilation. The resulting reduction in serum bicarbonate can shift the patient from an alkalotic to acidotic state if pulmonary complications are present, and Sulfamylon should be stopped for 24-48 hours in this instance (3). Mafenide acetate is available as an easily transportable powder for reconstitution as a $5 \%$ aqueous solution but has the disadvantage of requiring regular attention to prevent drying out and becoming ineffective. Potentially hypothermia can develop when 'soaks' are applied, although this might be prevented by application beneath Gortex ${ }^{\circledR}$ or other breathable fabric garments (vide infra). Mafenide has also been used successfully in tropical environments as a spray, which is easier to apply and more economical (4). When used as an $1.5 \%$ cream in a water miscible base it acts as a constant release preparation, which does not require as much attention as 'soaks' and only need replacement every 24 hours like Flamazine.

\section{Do topical anti-microbial agents work?}

Large numbers of casualties with burn injuries will place a strain on logistical elements of a command structure, including provision of medical supplies. Current SOPs require the application of topical antimicrobial cream to all burn injuries, yet this may not be an effective utilization of resources. To ascertain which burn patients will receive benefit from the application of topical anti microbial agents, a comparison was made between mortality in burn patients pre-mafenide use (1950-1963) with data from the period when mafenide was routinely employed (1964-1968), but in all other respects treatment was identical (5). For patients of combatant age, the benefit is only minor on burns less than $40 \%$ total body surface area (TBSA), and is maximal for those with burns of $60 \%$ TBSA. The data demonstrate two truisms; that small burns usually survive and large burns usually die, regardless of interventions such as the topical application of antimicrobials. Infection of very small or large burns does not appear to contribute significantly to mortality, although there may be a local effect in small burns not reflected in the data or addressed by this study. However, it would appear that mandatory application of burn creams to all burn injuries is an ineffective use of resources. Caution should be exercised in applying the conclusions of this historical study to present-day practice. These results do not define the relative effect of topical antimicrobial use in patients who enjoy all the other advances in burn care which have occurred since 1968, or the relative effect in a combat environment. However, it is reasonable to apply the conclusions to patients encountered on humanitarian operations who may never reach a burn unit or combatants experiencing significant delays in receiving treatment.

\section{Cerium nitrate}

Despite appropriate antibiotics and traditional dressings, some patients with large burns who are cardiovascularly stable and well resuscitated, succumb earlier than would be anticipated. Although infection at the interface between eschar and viable tissue may contribute a systemic effect of dead burnt tissue seems to be responsible for the demise of these patients. The ability of burned skin to kill in a dose dependant manner has been demonstrated in mice by injecting fractionated homogenates of burn eschar (6). A polymer of cell membrane lipid protein complex with a molecular mass of 3 $\mathrm{x} 10^{6}$ is responsible for the effect by binding viable $T$ helper subsets, causing an imbalance in the ratio of IL2, IL6 and TNF. The clinical picture is still one of multi organ failure known as systemic immune response syndrome or SIRS. Rodent experiments demonstrate that the degree of immune upset is quantitatively linked to the amount of burnt tissue present, and that sub lethal quantities can effectively suppress the immune system, making the animal prone to infection (7). For these reasons early excision of burn eschar has been advocated and is the single most important factor in reducing mortality burns over the last quarter century, particularly in patients over 50 years of age.

An ideal burn dressing would address the concerns over eschar both by preventing bacterial invasion and also inhibiting release of lipoprotein complex into the circulation. Cerium nitrate is a heavy metal compound that remains under investigation as such a topical burn therapeutic agent. Although it has some antiseptic properties, survival of patients treated with cerium nitrate is greater than would be expected from standard mortality data, and is close to the expected 
survival of patients who undergo early excision of burn eschar (8-12). It has been demonstrated that cerium has the ability to bind LPCs responsible for SIRS without binding to collagen or albumen in uninjured skin (13). A distinct survival advantage is demonstrated in rodent models following treatment with cerium nitrate, and in humans a survival benefit is conferred by a single cerium nitrate bath on arrival in a unit, followed by standard care (14).

Like silver nitrate, cerium nitrate solution is not an easy compound to administer and lacks some of the analgesic properties of silver sulphadiazene. A mixture of the two compounds (Flamacerium) is commercially available or can be manufactured simply, but it is only available in the UK on a named patient basis. Its application to the management of military burn casualites requires urgent investigation, as a proportion of these seriously injured patients may be subjected to a significant delay before reaching a tertiary centre and a single application of cerium containing compound may confer a significant survival advantage.

\section{Dressing materials}

When presented with military burn casualties, application of a dressing must require minimal time commitment from skilled staff and consume minimal resources, yet at the same time remain effective.

Conventional dressings consist of petroleum gel gauze, and an absorbant layer, usually of surgical gauze, gamgee and crepe. Dressings become rapidly sodden and require frequent attention. In addition they make wound inspection difficult and early signs of infection can go unheeded. There is evidence that bacterial growth thrives within the warm, moist environment of these dressings (15). A highly absorbent material (Exu-Dry®, Smith and Nephew Ltd) fashioned into pre-formed gloves and suits which are easy to apply and lighter than traditional burns dressings is available, and although it has no intrinsic antibacterial properties, merits investigation when combined with a topical antimicrobial.

Gortex ${ }^{\circledR}$ is semi-permeable membrane made from expanded PTFE, which is vapour permeable but does not allow transmission of bacteria. Laboratory studies demonstrate that the membrane is able to separate sterile from innoculated media both on culture and by electron microscopic study (16). When made into gloves for hand burns, there are good healing rates with low levels of infection (17). Maceration of the skin and collection of exudates within the Gortex ${ }^{\circledR}$ dressing is avoided unlike a simple polythene bag dressing that is popular amongst burns units in the UK. However, because water vapour passes through Gortex $\AA$, burns tend to dry out and addition of Flamazine cream prevents this problem (18). The gloves are comfortable and allow a good range of motion, and are less painful to apply than traditional dressings. Gortex ${ }^{\circledR}$ gloves are easy to fit and require little aftercare, with patients able to undertake the dressing themselves. Lower limb Gortex ${ }^{\circledR}$ garments have also been used successfully.

Gortex ${ }^{\circledR}$ is a plentiful material in the military. Its inclusion as the weather protection of choice for combatants demonstrates its durability. Soldier 95 combat uniform contains Gortex ${ }^{\circledR}$ gloves, which when coated on the inside with Flamazine or similar will produce a readily available dressing, superior to traditional materials. For larger burns the issue waterproofs potentially could be used to good effect. In major burns that require transport, the issue bivi bag could be employed. Utilizing elements of a soldier's uniform in combination with topical antimicrobials as a burn dressing reduces the drain on field installation, whilst potentially providing the patient with a superior medical treatment.

\section{Conclusions}

Burn injury is likely to be an increasingly common feature of modern warfare. Although the majority of surgery for these patients is likely to occur in tertiary centres, careful packaging of casualties is important to ensure that they have an optimal chance of recovery. Some relatively simple alterations to SOPs, based on good published evidence could substantially improve our management of these injuries.

\section{References}

1. Mason AD, McManus AT, Pruitt BA. Association of burn mortality and bacteraemia: a 25 year review. Archives of Surgery 1986: 121; 1027-1031.

2. Northern General Hospital, Dept of Microbiology 2001, personnel communication.

3. Pruitt BA Jr et al. The effect of Sulfamylon on the electrolyte pattern of the acute burn. US Army Research Unit Annual Progress Report 1967, BAMC, FSHT Sec 2.

4. Mendelson JA. The treatment of burns under conditions of limited resources using topical aqueous sulfamylon hydrochloride spray. Fournal of Burn Care and Rehabilitation 1997;18:238-244.

5. T P La Brown, Cancio L, Mason AD Jr., McManus AT, Goodwin CW. Survival benefit conferred by topical antimicrobial preparations in burn patients: Applicability of historical data to modern conflict. (in Press).

6. Schoenenberger GA, Bauer UR, Cueni LB, Eppenberger U, Allgower M. Isolation \& characterization of a cutaneous lipoprotein with lethal effects produced by thermal injury in mouse skin. Biochim Biophys Res Commun 1971;42:975.

7. Schoenenberger GA, Burkhardt F, Kalberer F et al. Experimental evidence for a significant impairment of host defense for gram-negative organisms by a specific cutaneous toxin produced by severe burn injuries. Surg Gynaecol Onstet 1075;141:555.

8. Monafo WW, Tandon SN, Ayvazian VH. Cerium nitrate: a new topical antiseptic for extensive burns. Surgery 1976;80:465.

9. Fox CC, Monafo WW, Ayvazian VH. Topical chemotherapy for burn using cerium salts and silver sulphadiazene. Surgery, Gynaecology and Obstetrics 1977;144:668. 
10. Monafo WW, Robinson HN, Yoshioka T. Lethal burns. Archives of Surgery 1978;113:397.

11. Kistler D, Hafermann B, Schoenberger GA et al. Increased survival rates by topical treatment of burns with ceruim nitrate. European Surgical Research 1990;22:283.

12. Schoenenberger GA, Kislter D, Klein $\mathrm{P}$ et al. Experimental comparison of the effect of early excision vs topical cerium nitrate upon late mortality with respect to cutaneous toxin absorption. European Surgical Research 1981;13:64.

13. Kistler D, Hafermann B, Schoenenberger GA, Hettich R. Increased survival rates by topical treatment of burns with cerium nitrate. Eur Surg Res 1990;22:283.

14. Scheidedder D, Sparkes BG, Lusder N et al. Survival in major burn injuries treatment by one bath in $\mathrm{Ce}\left(\mathrm{NO}_{3}\right)_{3}$. Burns 1992;18:296.

15. Drucker WR et al. In Surgery - a problem solving approach 2nd edition, 1995 Ed J H Davis and G F Sheldon Mosby, St Louis.

16. Martin DL, French GWG, Theakstone J. The use of semi permeable membranes for wound management. British Fournal of Plastic Surgery 1990:34;55-60.

17. Martin DL, Henley M. An approach to the management of hand burns using semi-permeable membranes. In The Management of Mass Burn Casualties and Fire Disasters Ed Masselis M, Gunn SWA Kluwer Academic Publishers, Netherlands 1993: 288-291.

18. Hoffman S. Silver sulphadiazene. An antibacterial agent for topical use in burns. Scandinavian. Fournal of Plastic Surgery 1984;8:119. 